\title{
Equation-Method with Enhanced Strategies for Reducing the Bit-errors Caused by Clipping Distortion in OFDM Signals
}

\author{
Nargis Bibi, Anthony Kleerekoper, Barry Cheetham, Nick Filer \\ School of Computer Science \\ The University of Manchester \\ Manchester, UK \\ Email: bibin@cs.man.ac.uk, kleereka@cs.man.ac.uk, barry@man.ac.uk,nick@man.ac.uk
}

\begin{abstract}
Orthogonal Frequency Division Multiplexing (OFDM) is the digital modulation technique used by 4G and many other communication systems. OFDM waveforms have high amplitude fluctuations resulting in high Peak to Average Power Ratios (PAPR). This can make an OFDM transmitter susceptible to non-linear distortion produced by its high power amplifiers (HPA). Limiting the peaks before an OFDM signal is applied to the HPA causes in-band distortion which introduces bit-errors at the receiver additional to those due to additive white Gaussian noise (AWGN). There have been many ideas for reducing the resulting bit-error rate. An 'Equation-Method' is now proposed, based directly on the fast Fourier transform (FFT). Clipped time-domain samples become the unknowns of a set of equations derived from the received constellation. The use of 'dither' and recursion at the transmitter allows the receiver to identify reliable frequency-domain symbols which may be used to formulate the equations. This approach is receiver oriented and the receiver is assumed to know the clipping levels. Four enhanced strategies are proposed in this paper for the Equation-Method. Simulation results are presented to show that the Equation-Method performs well with severe clipping as compared to other receiver-oriented techniques such as the Bussgang noise cancellation (BNC) iterative receiver.
\end{abstract}

Keywords-clipping; orthogonal frequency division multiplexing (OFDM); FFT; peak to average power ratio (PAPR) ; pinv ; dither; recursion

\section{INTRODUCTION}

Orthogonal Frequency Division Multiplexing (OFDM) is a multicarrier modulation technique which is being used in many wired and wireless systems including Asymmetric Digital Subscriber Line (ADSL), Digital Audio/Video Broadcasting (DAB/DVB), LTE and WiMAX standards. The main advantage of OFDM over conventional single carrier systems is its spectral efficiency and invulnerability to multipath effects. One of the major drawbacks to the practical implementation of OFDM systems is its relatively high peak to average power ratios (PAPR). The high peaks can produce serious in-band nonlinear distortion when applied to the High Power Amplifier (HPA) at the transmitter. It requires a highly linear HPA with large backoff and a large dynamic range of digital to analog converters (DAC). The PAPR is more important for uplink transmissions as it is more expensive in terms of power consumption for a mobile terminal to afford a linear amplifier.

In the literature, there are two types of methods to solve this problem. The first one is to generate only OFDM symbols with low PAPR, and the second one is to allow distortion to occur and then mitigate its effects at the receiver. There are some hybrid techniques which combine the properties of both types of methods. The most prominent among these are soft-clipping and filtering, coding, selected mapping and partial transmit sequences [1]-[5]. With $\mathrm{N}$ subcarriers per OFDM symbol, the peak power can theoretically be $\mathrm{N}$ times the average power. Keeping in mind the cost and implementation of a linear HPA it is preferable to limit the signal at the maximum permissible amplitude [6]. The highest peaks occur when all the subcarriers are modulated using same phase and also when the phases alternate.

Soft-clipping is a simple technique for reducing PAPR. The time-domain signal is passed through a soft-limiter in the transmitter to reduce the amplitudes of the large peaks to a predefined threshold, before it is applied to the amplifier. The clipping is a non-linear process which degrades the performance at the receiver resulting in an increase in biterror rate (BER). Sampling at the Nyquist sampling rate causes all the clipping noise to fall in-band and there is significant re-growth of peaks after digital to analog D/A conversion. It has been shown in [6], [7] that clipping an oversampled signal reduces peak power re-growth and, also, less distortion occurs in-band. The clipping introduces both in-band and out-of-band (OOB) distortion. The OOB may be removed by applying filtering. There are a few methods in the literature to compensate for the effect of the in-band clipping at the receiver to reduce BER. Iterative receivers such as Bussgang noise cancellation (BNC) and decision aided reconstruction (DAR) can reduce clipping noise effects at the receiver and ultimately reduce bit-errors [8]-[10], but these methods use an extra pair of IFFT/FFT transforms which increase the computational complexity. 
[11] proposed a joint iterative channel estimation and clipping noise reduction technique which is based on the minimum mean square error (MMSE) criterion. Another research has focused on taking the clipping as rare event and hence evaluating and analyzing conditional probability of the bit-errors in such an event [12]. In [13], authors have proposed to copy the clipped part of the OFDM signal and provide it to the receiver to recover the original unclipped signal. There are approaches which instead of performing clipping at the transmitter, try to mitigate the effects of nonlinear distortions produced by the HPA [14].

This paper presents a new way of reducing the BER due to clipping. It is referred to as the 'Equation-Method'. A description of the proposed method is presented here with an evaluation and comparison, in terms of BER reduction, with other approaches.

The rest of this paper is organized as follows: Section 2 describes an OFDM system transmitter. Section 3 explains soft limiting and filtering. Section 4 explains the methodology of the proposed Equation-Method, Section 5 presents simulation results. Section 6 discusses the results in comparison to the other approaches and Section 7 of the paper draws some conclusions.

\section{OFDM SYSTEM MODEL}

A baseband OFDM signal is expressed as:

$$
x(n)=\frac{1}{\sqrt{N}} \sum_{k=0}^{N-1} X_{k} e^{\frac{j 2 \pi n k}{N}} \quad 0 \leq n<N-1
$$

where $N$ is the number of subcarriers and $X_{k}$ for $k=0,1, \ldots, N-1$ are the complex modulated data symbols. Any cyclic extension is disregarded.

The PAPR of the OFDM symbol is defined as the ratio of the maximum to the average power of the signal. Mathematically it can be written as:

$$
P A P R=\frac{\max _{0 \leq n<N-1}|x(n)|^{2}}{E\left\{|x(n)|^{2}\right\}}
$$

Problems occur because of high peaks which fall in the non-linear range of the amplifier. This results in loss of information at the receiver increasing the BER.

\section{CLIPPING}

OFDM Signal in time-domain may be expressed in polar form as:

$$
x_{n}=x(n) \cdot e^{j \theta(n)}=x_{I}(n)+j x_{Q}(n)
$$

where $x_{I}(n)$ and $x_{Q}(n)$ are the in-phase and quadrature components of the complex envelope in discrete form, respectively.

Clipping is performed in the time-domain as in [15]. The amplitudes of the time-domain samples are limited to predefined threshold $A$, keeping the phases unchanged. The clipping process is described by the following expression:

$$
x_{c}(n)=\left\{\begin{array}{ll}
x_{n}, & \text { if }\left|x_{n}\right| \leq A \\
A \cdot e^{j \theta(n)}, & \text { if }\left|x_{n}\right|>A
\end{array}\right\}
$$

where $A$ is the maximum permissible amplitude and $x_{c}(n)$ is the clipped signal.

The $x_{c}(n)$ samples are then passed through additive white Gaussian noise (AWGN) channel. We have assumed perfect carrier and timing synchronization of the receiver. In addition, the clipping is applied at the Nyquist sampling rate, hence the algorithm is developed to mitigate in-band noise only and the effect of OOB distortion will not be addressed in this paper.

\section{The Equation-Method}

The receiver applies the received clipped time-domain signal to an Nth order FFT and converts it into the frequency-domain expressed in matrix form as:

$$
\underline{Y}=P . \underline{X}
$$

where $\underline{X}$ and $\underline{Y}$ are the $N \times 1$ vectors of time-domain and frequency-domain samples respectively, and the elements of the $N \times N$ matrix $P$ are as follows:

$$
p(n, k)=\exp (-2 \times \pi \times j \times(n-1) \times(k-1) / N)
$$

This produces complex constellation symbols which may be mapped onto real and imaginary axes. The constellations for all possible modulation techniques (PSK, QPSK, 16QAM, etc.) are defined by known fixed positions on the complex plane. But since the signal has been clipped and also affected by channel noise (AWGN), the received constellation will be different from that of the transmitted signal. If the clipping distortion and channel noise are small, it is possible that the received symbols are close, in a Euclidean sense, to the fixed constellation points. In this case, 'snapping' each symbol to the nearest fixed constellation point will recreate the original constellation at the transmitter, and no bit-errors at all will then be incurred.

TABLE I. ESTIMATE OF CLIPPED SAMPLES BEFORE ERRORCORRECTION

\begin{tabular}{|c|c|c|}
\hline A & $\begin{array}{c}\text { Max. No. of Clipped } \\
\text { Samples }\end{array}$ & $\begin{array}{c}\text { Min. No. of Clipped } \\
\text { Samples }\end{array}$ \\
\hline 0.75 & 7 & 0 \\
\hline 0.70 & 9 & 0 \\
\hline 0.65 & 10 & 1 \\
\hline 0.60 & 14 & 2 \\
\hline 0.55 & 18 & 4 \\
\hline 0.50 & 22 & 9 \\
\hline 0.45 & 26 & 13 \\
\hline 0.4 & 30 & \\
\hline
\end{tabular}

In more interesting cases where clipping distortion and/or channel noise cause some received symbols to move further away from the true constellation points, bit-errors are created when these received symbols are snapped to incorrect constellation points. The Equation-Method aims to use the information provided by correctly snapped symbols to correct clipping distortion in the time-domain, where the 
majority of the symbols received are correctly snapped, this is quite straightforward as illustrated by the data in Table I. Table I gives the details about maximum and minimum number of clipped samples (out of 64 samples) with different clipping thresholds. It is evident from the results that even at severe clipping, there are enough unclipped correct samples may be used to generate equations.

Firstly, the receiver must decide which time-domain samples have been clipped which is reasonably straightforward when the channel noise is negligible and the clipping level A is known at the receiver. We consider this case first, and disregard the effect of making errors in this classification.

At the receiver, the received time-domain samples are converted to frequency-domain complex symbols and produced $\underline{Y}$, which are snapped to the fixed constellation points. If we know exactly that the samples which have been clipped, and the frequency-domain samples which are correctly snapped and did not get affected by clipping, the FFT equation 5 may be partitioned as:

$$
\left[\begin{array}{l}
\underline{Y}_{R} \\
\underline{Y}_{U}
\end{array}\right]=\left[\begin{array}{ll}
G & F \\
E & D
\end{array}\right] \times\left[\begin{array}{l}
\underline{X}_{C} \\
\underline{x}_{O}
\end{array}\right]
$$

where the vector $\underline{Y}$ is partitioned as $\underline{Y}_{R}$ representing reliable frequency-domain symbols and $\underline{Y}_{U}$ giving unreliable symbols. Time-domain vector $\underline{x}$ is divided in two sub-matrices as the set of clipped sampled $\underline{x}_{c}$ and nonclipped samples $\underline{x}_{o}$ respectively. The matrix $P$ is partitioned such that $G$ and $E$ are matrices of the sinusoidal coefficients associated with the time-domain samples $\underline{x}_{c}$, whereas $F$ and $D$ are matrices of the sinusoidal coefficients associated with the $\underline{x}_{o}$ respectively. Furthermore, the sub-matrices $G$ and $F$ correspond to the reliable frequency-domain symbols $\underline{Y}_{R}$, whereas $E$ and $D$ correspond to the unreliable frequency domain symbols $\underline{Y}_{U}$.

Equation 7 represents the case when we have the true knowledge of the clipped samples and correct frequencydomain symbols. In the real scenario, after receiving the clipped signal, the OFDM signal is converted to the frequency-domain symbols and snapping the constellation symbols produce $\underline{\hat{Y}}$.

Dividing the received complex time-domain samples $x(n)$ for $n=1,2, \ldots N$, into clipped samples $x_{c}(n)$ for $n=1, . . L$ and non-clipped samples (the others) $x_{o}(n)$ for $n=1, . ., N-L$ allows the FFT Equation 5 at the receiver to be partitioned as follows:

$$
\left[\begin{array}{l}
\hat{\underline{Y}}_{R} \\
\hat{\underline{Y}}_{U}
\end{array}\right]=\left[\begin{array}{ll}
G & F \\
E & D
\end{array}\right] \times\left[\begin{array}{l}
\hat{x}_{c} \\
\underline{x}_{o}
\end{array}\right]
$$

In equation 8 , the frequency domain symbols $\underline{\hat{Y}}$ have also been partitioned according to whether they are considered likely to be reliable $\underline{\hat{Y}}_{R}$ or unreliable $\underline{\hat{Y}}_{U}$, after they are snapped to the nearest constellation point. The partitioning of $\underline{\hat{Y}}$ becomes a crucial aspect of this method and is discussed later in details. The dimensions of $\hat{\underline{Y}}_{R}$ and $\hat{\underline{Y}}_{U}$ are defined as $M \times 1$ and $(N-M) \times 1$ respectively.

$$
\underline{\hat{Y}}_{R}=G \times \underline{\hat{x}}_{C}+F \times \underline{x}_{O}
$$

which means that when $L=M$ and $G$ is non-singular, the clipped time-domain samples now treated as unknowns, may be obtained as follows:

$$
\underline{\hat{x}}_{c}=G^{-1}\left(\underline{\hat{Y}}_{R}-F \times \underline{x}_{o}\right)
$$

Therefore, the true values of the clipped time-domain samples may be obtained from a knowledge of the other time-domain samples and the frequency-domain samples considered reliable when the latter are correctly snapped in the constellation.

When $M=L$, the resulting square matrix $G$ is not guaranteed to be non-singular. Fortunately, the case where $M=L$ is not the most interesting. When $M>L$, matrix $G$ becomes non-square (i.e. 'thin') and Equation 9 becomes over-determined with more linear equations than variables. Now, provided the rank of $G$ is not less than $L$, the 'pseudoinverse' solution to Equation 10:

$$
\underline{\hat{x}}_{c}=G^{\#}\left(\underline{\hat{Y}}_{R}-F \times \underline{x}_{o}\right)
$$

Equation 11 gives a set of unknown time-domain samples $\underline{\hat{x}}_{C}$ that come as close as possible to minimizing the difference between the left-hand and right-hand sides of Equation 9 in a 'least sum of squares' sense. $G^{\#}$ is the lefthanded pseudo-inverse of $G$ as would be calculated by the MATLAB function 'pinv'.

Where the time-domain clipping and channel noise are not too severe, and therefore many frequency-domain symbols are correctly snapped, the pseudo inverse-solution becomes relatively insensitive to the choice of $M$ and 'reliable' symbols at the receiver. If some incorrectly snapped symbols are inadvertently included in the set considered reliable, their effect becomes overwhelmed by the correctly snapped symbols in the pseudo-inverse solution. Without a method of deciding which frequency-domain symbols are reliable, the receiver may take all of them, i.e. making $M=N$, assuming that the large majority will be reliable. This naive approach works reasonably well in conditions of low distortion and channel noise.

A better strategy for the receiver is to choose as 'likely to be reliable' those symbols which are closest (in a Euclidean sense) to the fixed PSK, QPSK or QAM constellation points. This works better, but requires a choice of 'threshold of closeness' and will make mistakes when larger distortion or channel noise drives a symbol well away from the correct constellation point and into the vicinity of a different one. To make the Equation-Method more effective for higher levels of distortion and channel noise, a strategy may be introduced at the transmitter to help the receiver make appropriate 'reliability' decisions.

The further enhanced strategy is to introduce a degree of randomness or 'dither' into the original symbols at the transmitter and introduce a form of recursion which allows the transmitter to anticipate the decisions of the receiver. 
Firstly, each symbol at the receiver is perturbed by a small random vector but only in such a way that it is not likely to affect the snapping decision at the receiver. It is fundamental to this idea that because constellation points are effectively quantized (there is finite distance between all of them), a small amount of dither can be added to what is transmitted without this necessarily affecting the quantized complex numbers that define the constellation. Dither has been used in other work on PAPR mitigation, but for different reasons [16]. In the Equation-Method the nature of the dither is chosen to try to ensure that unreliable symbols can readily be identified as such at the receiver.

To implement this idea, a copy of the receiver is mirrored at the transmitter to allow the transmitter to monitor, approximately, the effect of a given choice of dither vectors on the likely decisions. The process is approximate because, although the effect of the dither can be anticipated, it is more difficult to anticipate the effect of the channel noise. In the current implementation, a random set of dither vectors of gradually increasing length is tested until one is found that moves the symbols in such a way that a 'minimum distance' strategy adopted at the receiver will identify the reliable and unreliable symbols. The minimum distance' strategy is simply to take the symbols closest to a constellation point as reliable, therefore the dither must move the unreliable ones away from constellation points. The mirrored receiver aims anticipates the effect of each choice of dither vectors.

The dither and recursion strategy moves constellation symbols which are wrongly going to be selected as reliable further away from the wrong fixed constellation points. Computational savings may be made by applying dither selectively to these symbols only, thus allowing a more efficient search strategy.

\section{RESULTS AND DISCUSSION}

This section investigates the performance of the proposed algorithm by the MATLAB simulation of an OFDM system with 64 sub-carriers and a 16-QAM constellation on each sub-carrier. First we assess the effect of clipping when there is no bit-error correction. We have used the Moore-Penrose pseudo-inverse (pinv) function [17] as provided by MATLAB.

\section{A. Effect of clipping with no bit-error correction}

Fig. 1 plots estimates of the probability that the number, $N_{c S}$, of correctly snapped constellation symbols in an OFDM symbol at the receiver exceeds a given number, $C_{o}$, over the range 0 to 64 , when there is no attempt at symbol-error correction. Each estimate corresponds to a different value of the clipping threshold $A$ over a range from 0.4 to 0.65 . The average power of the OFDM signal, estimated over 10,000 OFDM symbols, is 0.156 , therefore the clipping ratio (CR) varies from approximately 1.01 , when $A=0.4$ to 1.64 when $A=0.65$. The Clipping ratio $(\mathrm{CR})$ is defined as the ratio of the clipping threshold $A$ to the root mean square (RMS) value $\sigma$ of the OFDM signal. The range therefore encompasses mild to very severe clipping.

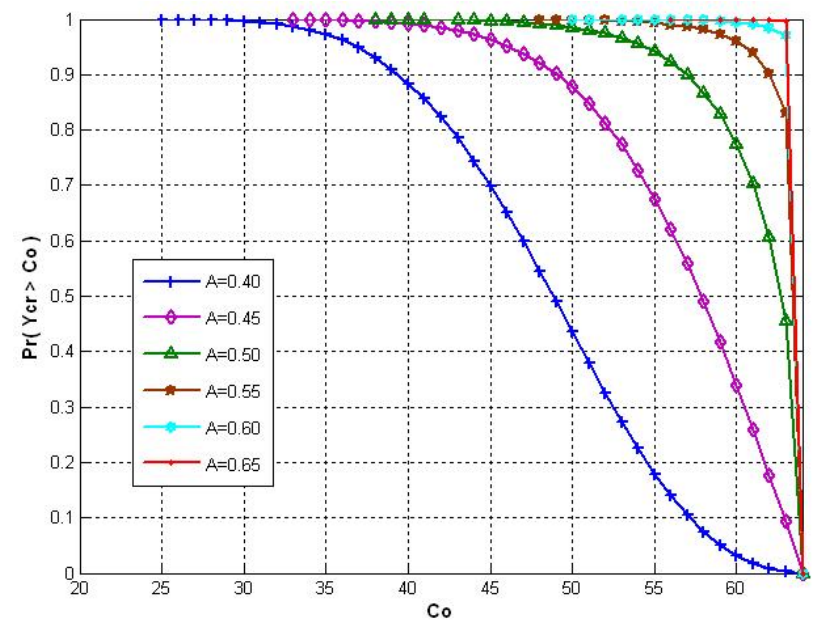

Figure 1. Distribution of $N_{c s}$ with no symbol-error correction

The probability estimates were produced from 10,000 randomly generated OFDM symbols for each selection of clipping threshold $A$. The plots show that even with very severe clipping $(A=0.4)$ and no error correction, there is still a reasonably high probability that more than half of the constellation symbols are correct, though the probability of having all symbols correct is low even for mild clipping. The red curve plotted in Fig. 2 shows the probability of an OFDM symbol error for different values of $A$. An OFDM symbol is error-free only when all constellation symbols are correct, and therefore all bits, are received correctly. Without symbol-error correction, the OFDM symbol error rate is seen to increase rapidly as the clipping threshold decreases.

\section{B. Equation-Method with $M=N$}

The simulations carried out for the zero symbol-error correction case were repeated with the proposed EquationMethod implemented with the number of equations $M$ equal to the number of sub-carriers, i.e. 64 . The number $L$ of clipped time-domain samples, i.e. the number of unknowns, depends on the value of clipping threshold $A$. The blue curve in Fig. 2 shows the results of this implementation of the Equation-Method for different clipping thresholds. Comparing with the red curve, it can be seen that even this naive version of the Equation-Method is effective in reducing the OFDM symbol error rate. At the quite severe clipping level $A=0.45$, the receiver correctly receives (after correction) about $50 \%$ of the transmitted OFDM symbols, where less than $10 \%$ would be received correctly without symbol-error correction. Taking $M=N$ means that wrongly snapped constellation symbols are included in the $M$ equations, and it would clearly be beneficial to exclude some or all of these. 


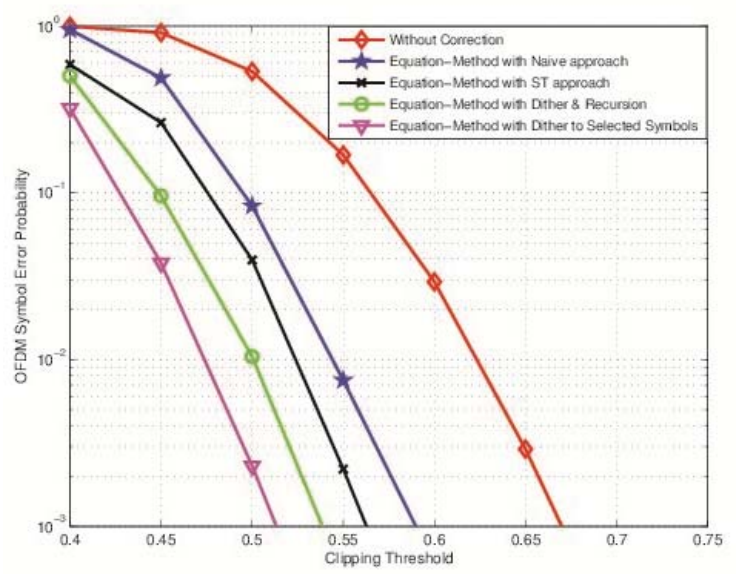

Figure 2. Symbol error Probability using Equation-Method

\section{Equation-Method with Optimized Snapping Threshold}

The 'Naive' strategy for the receiver can be improved by choosing, as 'likely to be reliable' those symbols which are closest (in a Euclidean sense) to the fixed PSK, QPSK or QAM constellation points. This new strategy requires a choice of distance threshold which we call the 'snapping threshold' (ST). The constellation symbols which move less than the ST distance when 'snapped' to the standard constellation points are considered as reliable constellation symbols and are chosen to generate the equations. This is the mechanism by which the receiver makes its reliability decisions.

This version aims to exclude unreliable constellation symbols from Equation 11 by setting a snapping threshold (ST) distance. Now the receiver only includes those symbols which have moved less than ST on the constellation in the process of snapping from the received symbol to a valid constellation point. The effectiveness of this strategy varies with the choice of ST and the results presented here are for optimized values of ST. The black curve in Fig. 2 plots the symbol error probability against clipping threshold and may be compared with the previous curves. There is a significant improvement over the Naive method. For the severe clipping threshold $A=0.40$, the receiver now correctly receives OFDM symbols with a probability of about 0.4 , which means that $40 \%$ will be correct as compared with $5 \%$ for the earlier naive strategy $(M=N)$ and $0.25 \%$ without symbolerror correction.

\section{Equation-Method with Dither and Recursion}

This is an enhanced strategy for the Equation-Method which introduces a degree of randomness or 'dither' into the original symbols at the transmitter. It also introduces a form of recursion which allows the transmitter to anticipate the decisions of the receiver. Recursion means transmitter has a built-in copy of the receiver and its functions. The dither is introduced to try to ensure that the snapping threshold distance method described above works, and the mirrored receiver allows the appropriate dither vectors to be determined.

1) Dither: Firstly, each symbol at the mirrored receiver is perturbed by a small random vector but only in such a way that it is not likely to affect the snapping decision at the receiver. It is fundamental to this idea that because constellation points are effectively quantized (there is finite distance between all of them), a small amount of dither can be added to what is transmitted without this necessarily affecting the quantized complex numbers that define the constellation. Dither has been used in other work on PAPR mitigation, but for different reasons [16]. In the EquationMethod the nature of the dither is chosen to try to ensure that unreliable symbols can readily be identified as such at the receiver. To implement this idea, a copy of the receiver is mirrored at the transmitter to allow the transmitter to monitor, approximately, the effect of a given choice of dither vectors on the likely decisions. The process is approximate because, although the effect of the dither can be anticipated, it is more difficult to anticipate the effect of the channel noise. In the current implementation, a random set of dither vectors of gradually increasing length is tested until one is found that moves the symbols in such a way that a 'minimum distance' strategy adopted at the receiver will identify the reliable and unreliable symbols. The 'minimum distance' strategy is simply to take the symbols closest to a constellation point as reliable, therefore the dither must move the unreliable ones away from constellation points. The mirrored receiver aims to anticipate the effect of each choice of dither vectors.

2) Recursion: The transmitter has a mirror copy of the receiver so that it may know the behaviour of the receiver. According to the behaviour of the mirrored receiver, transmitter decides which copy of the signal needs to be transmitted. This is named as Recursion. It includes the following steps:

1) The mirror receiver converts the clipped baseband signal to the frequency-domain by applying FFT.

2) The frequency-domain constellation symbols are snapped to the fixed constellation points by keeping a record of the distance every constellation symbol moves.

3) The distances of the constellation symbols are sorted and the index of every constellation symbol is stored.

4) Now it has to choose reliable constellation symbols in order to write equations for them. The reliability will be decided on the basis of snapping threshold. The snapping threshold is fixed and is dependent on the clipping threshold. The array of distances is compared to the snapping threshold. All those constellation symbols which move less than the defined snapping threshold, will be separated in a matrix and rest of them are considered unreliable. 
5) Since this receiver is built-in at the transmitter. It has a copy of the original constellation symbols. It compares the new matrix of reliable constellation symbols with the original ones to find out if any of the chosen constellation symbols is wrong. It returns an array of wrongly chosen constellation symbols.

6) If the array of wrongly chosen constellation symbols is greater than 0 , then it sends the control to the dither function.

3) Results: Fig. 2 shows the probability of an OFDM symbol error for different values of A using the Dither and Recursion method. An OFDM symbol is error-free only when all constellation symbols are correct, and therefore all bits, are received correctly.

As before the results are presented for optimized ST thresholds. Now the probability of a correct OFDM symbol approaches $90 \%$ for a clipping threshold of $A=0.45$. Further improvement to the Equation Method can correct more OFDM symbols to avoid any retransmissions or other complexities. Introducing dither and recursion with a mirrored receiver at the transmitter has been implemented as described in Section V-D1.

All the 64 constellation symbols for an OFDM symbol are randomly dithered until the mirrored receiver confirms that only reliable symbols will be chosen for the $M$ equations. A maximum of 100 randomized dither vectors are tried. If the mirrored receiver is unable to identify an ideal case, then it transmits the original OFDM symbol to the receiver. Fig. 2 shows the effect of applying this version of the Equation-Method.

\section{E. Equation-Method with Selective Dither and recursion}

Applying dither to all the constellation symbols increases the complexity at the transmitter. The main purpose of applying Dither is to move those constellation symbols, which are wrongly going to be selected as reliable at the real receiver, away from the fixed constellation points. If we observe the original signal generated, there are few symbols which the recursion returns to be wrongly chosen at the real receiver. There is a possibility that dither may be applied to these symbols only instead of applying to all of them.

Dither is applied to selected symbols according to the following algorithm:

1) Complex constellation symbols are converted to time-domain by applying IFFT:

$$
x(n)=\frac{1}{\sqrt{N}} \sum_{k=0}^{N-1} X_{k} e^{\frac{j 2 \pi n k}{N}} \quad 0 \leq n<N-1
$$

2) The time-domain signal is applied to soft-clipping.

3) The clipped signal is passed to the Recursion block at the transmitter as described in Section V-D2.

4) If any of the constellation symbols are wrongly being considered as reliable, they are stored in an array $W$. The array $W$ is passed to the Dither function.

5) The loop of Dither runs only for length of the wrongly chosen symbols array $W$. A random small complex number is generated and added to these constellation symbols in the array $W$.

6) The new modified constellation symbols are converted to time-domain base-band signal and passed through soft-clipping.

7) The control of the program is passed to the recursion process as described in Section V-D2.

8) The loop of dither and recursion carries on for a number of iterations. Every new set of dithered constellation symbols are applied to recursion to see the feedback of the receiver. The best case will be when the array $W$ is empty.

1) Results: The OFDM symbol in first iteration is passed through the Recursion process. If the array of wrongly chosen constellation symbols $W$ is not empty, then the process of Selective Dither starts. After first iteration of Selective Dither and Recursion, a copy of array $W$ is stored in memory as $W_{b}$. The constellation symbols from the array $W$ are dithered until the mirrored receiver returns the $W$ empty. After every iteration of Dither and Recursion, the latest array $W$ is compared to the stored array $W_{b}$. The array with minimum size is stored as $W_{b}$. If the mirrored receiver is unable to find a best case when $W$ is empty, then it transmits the $W_{b}$, which will have least number of constellation symbols, which will be chosen as reliable at the real receiver.

Fig. 2 shows the OFDM symbol error probability using Selective Dither and Recursion method. It is compared to all earlier versions of the Equation-Method. The probability of a correct OFDM symbol approaches $68 \%$ for a clipping threshold of $A=0.4$. This is much better than all previously discussed results.

\section{DISCUSSION}

We have compared the best results of Equation-Method with other receiver-oriented approaches, such as the BNC iterative receiver, at moderate and severe clipping thresholds in the absence of AWGN noise.

Fig. 3 shows the performance of the Equation-Method with Selective Dither and Recursion in comparison to the BNC receiver. The OFDM symbol error probability for the Equation-Method (Selective Dither and Recursion) at clipping threshold $A=0.5$ is 0.02 , and using $\mathrm{BNC}$ receiver it is 0.04. The Equation-method has low symbol error probability as compared to $\mathrm{BNC}$ receiver for all the clipping thresholds. 


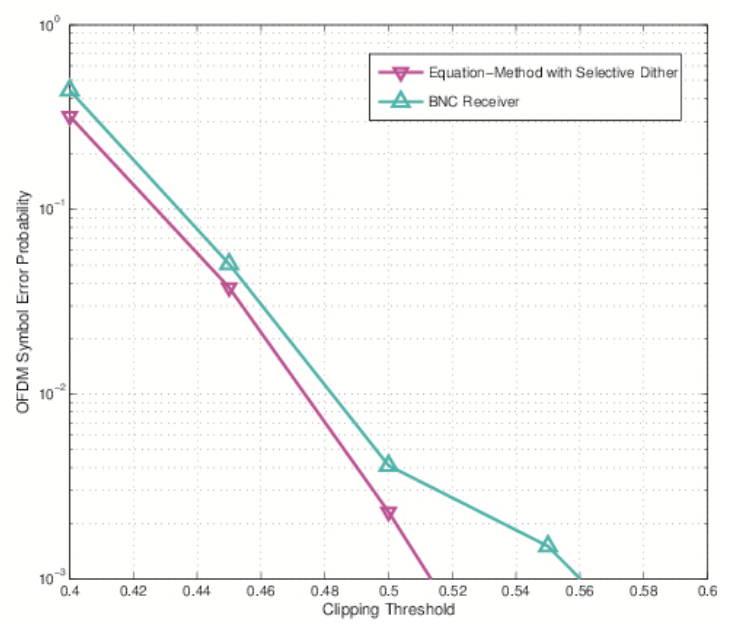

Figure 3. Comparison of the Equation-Method with BNC

\section{CONCLUSIONS}

A new method has been proposed for correcting the biterrors produced by clipping distortion in OFDM signals. It has been evaluated to determine the percentage of OFDM symbols that can be completely corrected, at moderate to severe clipping thresholds, by different versions of the method. In this paper, four strategies have been proposed to work with the Equation-Method. The Selective Dither and Recursion shows the best performance and correct majority of the received OFDM symbols completely at severe clipping threshold. The best results are compared with other receiver-oriented approaches, and demonstrate the superiority of the new method. The performance of the Equation-Method in the presence of AWGN will be addressed in future work.

\section{REFERENCES}

[1] T. Jiang and Y. Wu, "An overview: Peak-to-average power ratio reduction techniques for ofdm signals," IEEE Transactions on Broadcasting, vol. 54, no. 2, pp. 257-268, 2008.

[2] M. A. Taher, J. Mandeep, M. Ismail, S. A. Samad, and M. Islam, "Reducing the power envelope fluctuation of OFDM systems using side information supported amplitude clipping approach," International Journal of Circuit Theory and Applications, 2012.

[3] C.-E. Weng, C.-W. Chang, C.-H. Chen, and H.-L. Hung, "Novel lowcomplexity partial transmit sequences scheme for papr reduction in ofdm systems using adaptive differential evolution algorithm," Wireless Personal Communications, vol. 71, no. 1, pp. 679-694, 2013.

[4] O. Daoud and O. Alani, "Reducing the papr by utilisation of the LDPC code," IET Communications, vol. 3, no. 4, pp. 520-529, 2009.

[5] L. K. Kaur et al., "Peak to average power ratio reduction of OFDM signal by combining clipping with discrete cosine transform-ii," Global Journal of Researches In Engineering, vol. 13, no. 6, 2013.

[6] H. Ochiai and H. Imai, "Performance analysis of deliberately clipped OFDM signals," IEEE Transactions on Communications, vol. 50, pp. 89-101, Jan. 2002.

[7] H. Ochiai and H. Imai, "On clipping for peak power reduction of OFDM signals," in IEEE Global Telecommunications Conference, vol. 2, pp. $731-735,2000$.

[8] H. Chen and A. Haimovich, "An iterative method to restore the performance of clipped and filtered OFDM signals," in IEEE International Conference on Communications, vol. 5, pp. 3438-3442, May 2003.

[9] D. Kim and G. L. Stuber, "Clipping noise mitigation for OFDM by decision aided reconstruction," IEEE Communications Letters, vol. 3, pp. 4-6, 1999.

[10] N. Bibi and B. Cheetham, "Clipping noise mitigating iterative receivers for ofdm," in IEEE International Conference on Networked Embedded Systems for Every Application (NESEA), pp. 1-5, 2012.

[11] H. Gacanin and F. Adachi, "Iterative decision-directed estimation and compensation of nonlinear distortion effects for OFDM systems," Wireless Communications and Mobile Computing, vol. 12, no. 17, pp. 1558-1566, 2012.

[12] A. R. Bahai, M. Singh, A. J. Goldsmith, and B. Saltzberg, "A new approach for evaluating clipping distortion in multicarrier systems," Selected Areas in Communications, IEEE Journal on, vol. 20, no. 5, pp. 1037-1046, 2002.

[13] I. I. Al-kebsi, M. Ismail, K. Jumari, and T. Rahman, "Eliminate the effects of clipping technique on the ser performance by recovering the clipped part of the ofdm signal," International Journal of Computer Science and Network Security, vol. 9, no. 7, pp. 37-45, 2009.

[14] D. S. Owodunni, A. Ali, A. A. Quadeer, E. B. Al-Safadi, O. Hammi, and T. Y. Al-Naffouri, "Compressed sensing techniques for receiver based post-compensation of transmitter's nonlinear distortions in ofdm systems," Signal Processing, vol. 97, pp. 282-293, 2014.

[15] J. Armstrong, "Peak-to-average power reduction for OFDM by repeated clipping and frequency domain filtering," Electronics Letters, vol. 38, no. 5, pp. 246-247, 2002.

[16] K. Bae, J. G. Andrews, and E. J. Powers, "Adaptive active constellation extension algorithm for peak-to-average ratio reduction in OFDM," IEEE Communications Letters, vol. 14, no. 1, pp. 39-41, 2010.

[17] R. Penrose, "On best approximate solutions of linear matrix equations," in Proceedings of the Cambridge Philosophical Society, vol. 52, pp. 17-19, Cambridge Univ Press, 1956. 